\title{
Transformation: how do nematode sperm become activated and crawl?
}

\author{
Xuan Ma ${ }^{1}$, Yanmei Zhao ${ }^{1}$, Wei Sun ${ }^{1,2}$, Katsuya Shimabukuro ${ }^{\circledR}$, Long Miao ${ }^{1 凶}$ \\ ${ }^{1}$ Laboratory of Noncoding RNA, Institute of Biophysics, Chinese Academy of Sciences, Beijing 100101, China \\ ${ }^{2}$ Graduate University of Chinese Academy of Sciences, Beijing 100049, China \\ ${ }^{3}$ Department of Chemical and Biological Engineering, Ube National College of Technology, Ube, Yamaguchi 755-8555, Japan \\ $\triangle$ Correspondence: Imiao@moon.ibp.ac.cn (L. Miao); kshimabu@ube-k.ac.jp (K. Shimabukuro) \\ Received April 18, 2012 Accepted May 9, 2012
}

\begin{abstract}
Nematode sperm undergo a drastic physiological change during spermiogenesis (sperm activation). Unlike mammalian flagellated sperm, nematode sperm are amoeboid cells and their motility is driven by the dynamics of a cytoskeleton composed of major sperm protein (MSP) rather than actin found in other crawling cells. This review focuses on sperm from Caenorhabditis elegans and Ascaris suum to address the roles of external and internal factors that trigger sperm activation and power sperm motility. Nematode sperm can be activated in vitro by several factors, including Pronase and ionophores, and in vivo through the TRY-5 and SPE-8 pathways. Moreover, protease and protease inhibitors are crucial regulators of sperm maturation. MSP-based sperm motility involves a coupled process of protrusion and retraction, both of which have been reconstituted in vitro. Sperm motility is mediated by phosphorylation signals, as illustrated by identification of several key components (MPOP, MFPs and MPAK) in Ascaris and the characterization of GSP-3/4 in C. elegans.
\end{abstract}

KEYWORDS spermiogenesis, major sperm protein, sperm motility

\section{INTRODUCTION}

Fertilization is achieved by the fusion of a mature sperm with an oocyte to produce a diploid zygote. In nematode species, the primary sperm cells undergo spermatogenesis to produce spermatids, which remain in a non-motile state and are stored in the seminal vesicles. After ejaculation, spermatids are activated in the uterus through a process called spermiogenesis, and become motile spermatozoa. Spermatozoa are responsive to the chemotactic signals derived from the oocyte (Kubagawa et al., 2006; Edmonds et al., 2011) and are competent for fertilization with oocytes. Here we review two processes, spermiogenesis and major sperm protein (MSP)-based sperm motility, in the nematode species Caenorhabditis elegans and Ascaris suum (hereafter Ascaris).

\section{NEMATODE SPERM CAN BE ACTIVATED BY DIVERSE FACTORS}

C. elegans and Ascaris have an evolutionary divergence of 350 million years (Vanfleteren et al., 1994). C. elegans is androdioecious; i.e., it has two sexes, hermaphrodite and male. The hermaphrodite form is able to produce self-progeny but, when mated with a male his sperm are used preferentially for fertilization, producing out-crossed sexual progeny. In contrast, Ascaris is dioecious, being either male or female. In both species, the mature spermatids must sense extracellular signals to become activated, a critical step for sperm to become motile. During this process, sperm extend pseudopods driven by the assembly of a cytoskeleton composed of MSP, instead of actin, which more typically forms the basis of amoeboid cell motility. At this time, membranous organelles (MOs), a type of intracellular vesicle similar to lysosomes (Zhu et al., 2009), fuse with the plasma membrane and secrete specific components required for motility and fertilization (L'Hernault, 2006).

Early in vitro assays documented a wide range of factors that induce sperm activation in both species. In Ascaris, spermatids can be activated using trypsin, a-chymotrypsin, Streptomyces griseus protease, and vas deferens extract 
(Abbas and Cain, 1979; Sepsenwol and Taft, 1990). In contrast, $C$. elegans spermatids can be activated by Pronase, the cationic ionophore monensin, a weak base triethanolamine (TEA) and an anion channel inhibitor 4,4'-diisothiocyano-2,2'stilbenedisulfonic acid (DIDS) (Nelson and Ward, 1980; Ward et al., 1983; Shakes and Ward, 1989; Machaca et al., 1996). Pseudopod extension involves membrane rearrangement, during which ionic fluxes play essential roles. In C. elegans, sperm can be activated by blockage of the inward-rectifying chloride $\left(\mathrm{Clir}_{\text {ir }}\right.$ ) channels or by accumulation of $\mathrm{HCO}_{3}^{-}$in the cytoplasm, leading to an increase of intracellular $\mathrm{pH}$ (Machaca et al., 1996). In Ascaris, an optimal intracellular pH, possibly buffered by $\mathrm{HCO}_{3}^{-}$, is necessary for pseudopod integrity (Fraire-zamora and Cardullo, 2010). The cytosolic alkalization is associated with the exchange of protons with $\mathrm{Na}^{+}$and $\mathrm{K}^{+}$ions, as in the cases of monensin and valinomycin, both of which induce pseudopod extension (Nelson and Ward, 1980; Roberts and King, 1991). Extracellular $\mathrm{Ca}^{2+}$ was shown not to trigger sperm activation but three lines of evidences indicate intracellular $\mathrm{Ca}^{2+}$ is involved during spermiogenesis, e.g., in $\mathrm{C}$. elegans, the $\mathrm{Ca}^{2+}$-binding chaperone calreticulin (CRT-1) (Park et al., 2001), a $\mathrm{Ca}^{2+} /$ calmodulindependent protein phosphatase (PP2B) (Bandyopadhyay et al., 2002) and the ferlin protein (FER-1), which contains multiple calcium-sensing $\mathrm{C} 2$ domains (Washington and Ward, 2006), mediate the processes of pseudopod extension and MO fusion.

Molecular genetic studies of $C$. elegans mutants have identified key components that regulate spermiogenesis. SPE-8 pathway members (SPE-8, SPE-12, SPE-19, SPE-27 and SPE-29) are major players in the control of this process (Minniti et al., 1996; Nance et al., 1999; Nance et al., 2000; Geldziler et al., 2005; L'Hernault, 2009). The spe-8 group hermaphrodite mutant is self-sterile. However, when mated with wild type or spe-8 males, spe-8 partial loss-of-function (If) hermaphrodites can produce self-progeny. This phenomenon is called "transactivation". Different levels of transactivation have been observed, i.e. spe-8 hypomorphic mutants can be transactivated, whereas spe- 8 null mutants can rarely be transactivated (Nance et al., 1999). Transactivation is due to the transfer of the male activator in the seminal fluid to the hermaphrodite. TRY- 5 is a typical male activator (see later sections). It was proposed that the targets of TRY- 5 contain both non-SPE-8 group proteins (spe-8 partial If mutants can be fully transactivated) and SPE-8 group members (spe- 8 null mutants cannot be transactivated) (Smith and Stanfield, 2011). Transactivation is induced mostly via SPE-8 independent pathway, although SPE-8 group proteins retain some of their activities during this process.

SPE-6 and SPE-4 are two currently well-characterized suppressors of the SPE-8 pathway. SPE-6 is a casein I kinase (Varkey et al., 1993), whereas SPE-4 is a presenilin, an aspartyl protease that localizes within fibrous body-MOs (Arduengo et al., 1998). SPE-6 is thought to function down- stream of the SPE-8 pathway and the onset of spermiogenesis requires reduction of SPE-6 activity (L'Hernault, 2009; Nishimura and L'Hernault, 2010). SPE-4 may participate in proteolytic processing of SPE-8 group proteins to inhibit spermiogenesis (L'Hernault, 2009; Nishimura and L'Hernault, 2010).

Another crucial component found in the male reproductive tract, SWM-1, containing two trypsin inhibitor-like (TIL) domains, inhibits premature activation of sperm in the seminal vesicle. Premature sperm activation would lead to the failure of sperm transfer during insemination because of the adherent nature of spermatozoa. Thus, SWM-1 ensures male reproductive success by keeping sperm in a non-activated state in the male body (Singson, 2006; Stanfield and Villeneuve, 2006).

Recently our group and the group led by Standfield made intriguing progress in the identification of new components during sperm activation in Ascaris and C. elegans, respectively. Smith and Stanfield (Smith and Stanfield, 2011) identified a seminal fluid protease TRY- 5 as a sperm activator in $C$. elegans. They devised an elaborate model for sperm activation. In the male seminal vesicle, SWM-1 inhibits leaky TRY-5 to prevent sperm from activating and thus keeps seminal vesicle in an environment unfavorable for sperm activation. However, during ejaculation, the vas deferens secretes TRY-5 that induces sperm activation. In hermaphrodites, sperm activation can be triggered by an unknown factor, which is not TRY-5. When mating occurs, both the seminal fluid-derived TRY- 5 and the hermaphrodite activator are able to initiate sperm activation, a process dependent on SPE-8 signaling pathway (Fig. 1A). Our group reported comparable findings (Zhao et al., 2012). We performed extensive analyses on As_SRP-1, a serine protease inhibitor belonging to the serpin superfamily, originally localized in MOs of spermatids in Ascaris. We showed that As_SRP-1 released from activating spermatids, in trans, inhibits the activation of other surrounding spermatids, by blocking the vas deferens-derived sperm activator As_TRY-5 (a trypsin-like serine protease) through a suicide substrate mechanism of serpin. Meanwhile, As_SRP-1 functions in cis to support MSP-based cytoskeletal assembly in the spermatid that releases it, thereby facilitating sperm motility and enhancing the competitiveness of the resulting spermatozoon. These dual functions of As_SRP-1 facilitate the success of the activated sperm in sperm cooperation (in the male body to prevent premature activation) and sperm competition (in the female reproductive tract) (Fig. 1B). Sperm competition has been widely recognized as one of most potent driving forces of evolution. Previous studies on sperm competition mechanisms have focused on physical traits of the sperm and of the seminal fluid produced by several accessory glands in the male body. Our study demonstrates for the first time that, besides components secreted from accessory glands in male body, sperm also contributes a protein (As_SRP-1) to the seminal fluid, 
A

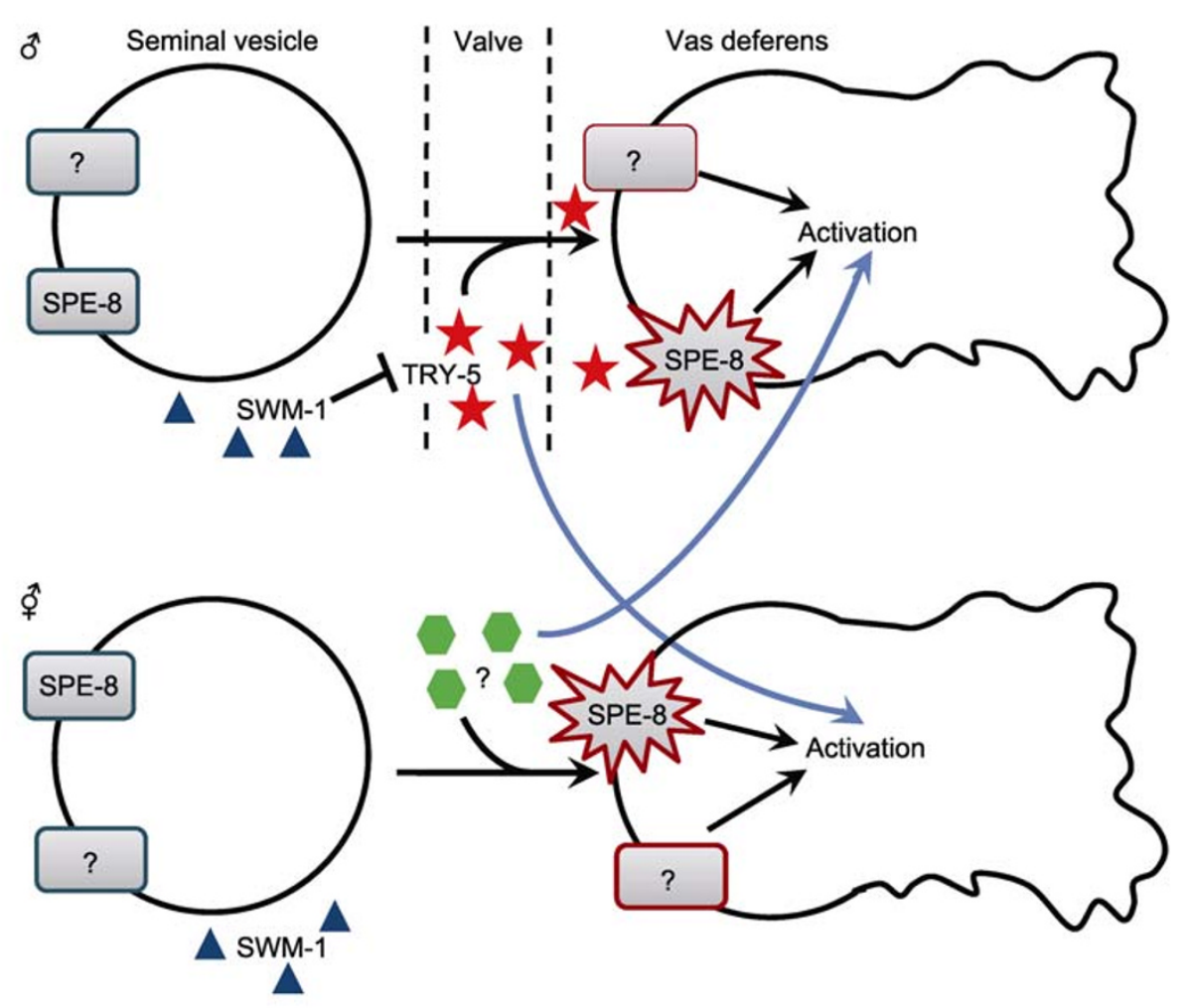

B

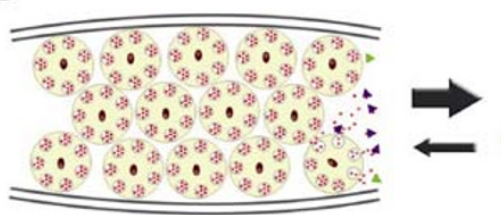

Seminal vesicle

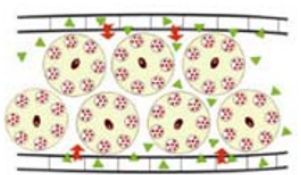

Vas deferens

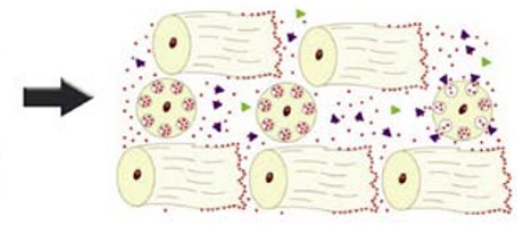

Uterus

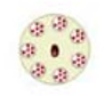

Spermatid a

MO
As_SRP-1

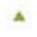

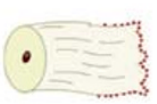

Figure 1. Models for the modulation of sperm activation by protease and protease inhibitors in $C$. elegans and Ascaris. (A) The end of $C$. elegans male gonad is composed of a seminal vesicle and vas deferens connected by a valve. The presence of SWM-1 (blue triangle) in the seminal vesicle inhibits leaky TRY-5 (red star) derived from vas deferens and prevents premature sperm activation. During the insemination, the high level of TRY- 5 secreted from the vas deferens is incorporated into seminal fluid and overcomes the inhibitory capacity of SWM-1, thus enabling sperm activation in the uterus via a SPE-8-independent pathway. The major targets of TRY-5 (rectangle with question mark) are unknown. Some receptors of TRY-5 may be SPE-8 group proteins. Blue and red rectangles indicate pre- and post-activated states. Hermaphrodite sperm activation is triggered by a currently unidentified factor (green hexagon) possibly solely via the SPE-8 pathway. When mating occurs, TRY-5 and the hermaphrodite activator are able to mutually activate both pathways, a phenomenon called "transactivations". (B) After the second meiosis and budding from the residual body, spherical inactive spermatids are stored in the seminal vesicle. When copulation occurs, the epithelia of the glandular vas deferens begin to secrete As_TRY-5 into the seminal fluid, causing a momentary increase of As_TRY-5 activity in the lumen. When passing through the narrow channel of the vas deferens, spermatids are exposed to and activated by As_TRY-5. The ensuing exocytosis releases the MO components, including non-membrane-bound As_SRP-1, into the seminal fluid. Sperm compete for activation and, later, for migration and fertilization. As_SRP-1 released from activated sperm inhibits As_TRY-5 in the seminal fluid by forming a covalent complex with it and thereby inhibiting activation of other spermatids. Meanwhile, As_SRP-1 associated with the inner leaflet of the MO membrane is displayed on the outer surface of spermatozoa following the fusion of MOs with the plasma membrane (PM). Alternatively, As_SRP-1 secreted into the seminal fluid could be recruited back to the cell surface of activated spermatozoa. In either case, we suggest that PM-bound As_SRP-1 facilitates MSP cytoskeleton dynamics and enhances sperm migration. When mating ends, the vas deferens stops secreting As_TRY- 5 and the residual As_TRY-5 in the male reproductive tract may trigger marginal sperm activation, and therefore the release of As_SRP-1. This, in turn, immediately extinguishes the residual As_TRY-5 activity. This negative feedback loop thus functions to maintain the male reproductive tract an unfavorable environment for sperm activation and to guarantee the efficiency of sperm transfer when mating takes place again. We propose that this 'yin-yang' relationship between the protease and the protease inhibitor in the seminal fluid modulates nematode sperm cooperation, motility and competition. 
and that this protein coordinates both spermatozoon motility and sperm competition. In summary, these studies provide evidence that protease(s) and protease inhibitor(s) coordinately modulate spermiogenesis. This mechanism may be conserved and extend to other species, e.g., $\sim 18 \%$ of the proteins in the Drosophila ejaculate are predicted to be proteases or protease inhibitors (Ram and Wolfner, 2007; Findlay et al., 2008), and the protease cascade acts during and following insemination (LaFlamme et al., 2012).

\section{MSP-BASED SPERM MOTILITY IS REGULATED BY PHOSPHORYLATION SIGNALING}

Once the nematode sperm are activated in the uterus, sperm motility becomes critical for male fertility. Because Ascaris sperm can easily be obtained in large quantities and are large, this species is suited for biochemical and biophysical studies of amoeboid sperm motility. Ascaris sperm utilize MSP, rather than actin, for locomotion, providing a simplified model for the study of amoeboid cell motility. The pioneering work of Italiano et al. (1996) established an in vitro system for MSP polymerization. By adding ATP to the cell-free sperm extract, MSP-based motility was reconstituted in vitro. Vesicles derived from the plasma membrane at the leading edge triggered the assembly of a columnar meshwork of MSP filaments called fibers, resembling the in vivo MSP polymerization at the leading edge during pseudopod protrusion. Later, Miao et al. (Miao et al., 2003) used the same cell-free extract to reconstitute in vitro the MSP cytoskeleton retraction, like that observed at the rear end of cells in vivo, by adding Yersinia tyrosine phosphatase (YOP). Because MSP lacks ATP-binding site and MSP filaments are non-polar, this work demonstrates that amoeboid cells can retract their posterior end and pull the cell body forward with no contractile input from conventional motor proteins such as myosin, and that protein phosphorylation/dephosphorylation functions as a molecular switch to modulate MSP cytoskeletal assembly and disassembly (Mogilner and Oster, 2003).

Biochemical studies identified several key proteins that regulate MSP cytoskeletal dynamics during amoeboid sperm motility. A 48-kDa MSP polymerization organizing protein (MPOP) was identified as the only membrane component that nucleates MSP assembly (LeClaire et al., 2003). Phosphorylation of MPOP is $\mathrm{pH}$-sensitive and seems to require a yet undetermined tyrosine kinase. Phospho-MPOP binds to and recruits a serine/threonine kinase, MPAK (MSP polymerization-activating kinase), to the membrane at the leading edge (Yi et al., 2007). MPAK phosphorylates MFP2 (MSP fiber protein 2), which in turn increases the rate of fiber growth. In contrast, MFP1 negatively regulates fiber elongation (Buttery et al., 2003). A putative protein phosphatase 2A (PP2A) is present in the cell body and targets MFP3 (Yi et al., 2009). Phosphorylated MFP3 binds to and stabilizes the MSP filaments, whereas, dephosphorylation of MFP3 by PP2A releases it from the cytoskeleton, leading to the disassembly of MSP fibers (Fig. 2).

Currently the sequence of MPOP has not been elucidated but the other Ascaris proteins all have homologs in $C$. elegans (PP2A: LET-92; MPAK: C39H3.1/Y38H8A.3; MFP3: SSQ1 to SSQ4; MFP2: ZK265.3; MFP1: MSD-4/SSP-9 to SSP-32). According to microarray analysis, all of these $C$. elegans homologous genes except for let-92 are highly enriched during spermatogenesis (Reinke et al., 2004). It was recently found that the $C$. elegans PP1 phosphatases GSP-3

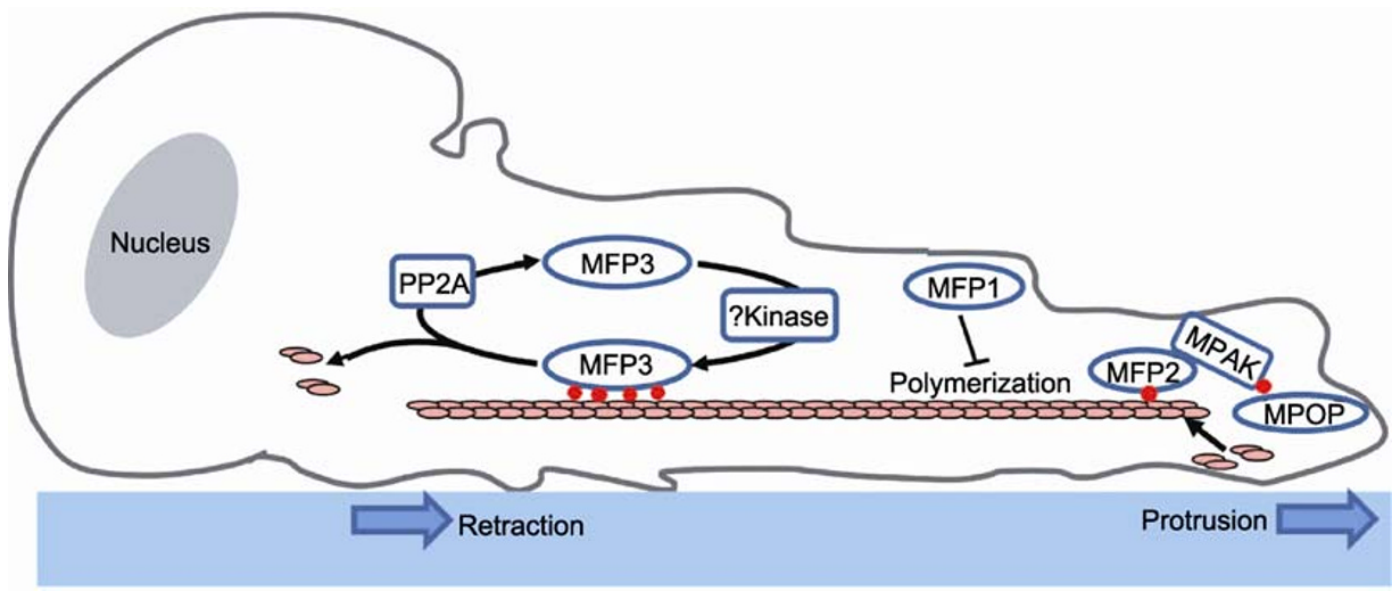

Figure 2. Schematic representation of MSP-based sperm motility in Ascaris. MSP dimers constitute the basic unit of the MSP fibers. MSP-based sperm motility is a coupled process of protrusion and retraction. At the leading edge, tyrosine phosphorylated MPOP recruits MPAK, which in turn phosphorylates MFP2. Phospho-MFP2 promotes MSP assembly, generating a protrusion force. MFP1 inhibits MSP polymerization. The cytosolic MFP3 is phosphorylated by an unknown kinase, and then binds to and stabilizes MSP filaments. At the cell body-pseudopod junction, PP2A becomes activated by a phosphatase and dephosphorylates phospho-MFP3, resulting in the release of MFP3 from MSP fibers. Ultimately, MSP fibers disassemble generating a retraction force near the cell body. 
and GSP-4 regulate MSP dynamics during sperm development (Wu et al., 2012). Lack of GSP-3/4 causes defects in pseudopod development and sperm motility. Taken together, these studies of both $C$. elegans and Ascaris are shedding lights on MSP-based amoeboid sperm motility.

\section{FUTURE PERSPECTIVES}

Nematode sperm provides a simplified system in which to study morphological and physiological changes associated with activation. In vitro assays can be readily conducted to examine the responses of sperm to various chemical compounds, such as ion carriers or ion channel inhibitors. These assays have demonstrated that ion channels (e.g., $\mathrm{Cl}^{-}, \mathrm{Na}^{+}$, and $\mathrm{K}^{+}$) that regulate intracellular $\mathrm{pH}$ are indispensable for nematode sperm activation. Through genetic screening, SPE-8 group proteins were shown to mediate sperm activation in both males and hermaphrodites. However, other pathways that respond to extracellular activators exist in both sexes. Early in vitro studies showed that a protease triggers sperm activation in Ascaris. Recent characterization of TRY-5 in C. elegans strongly supports the notion that the protease cleaves its receptors on the sperm surface to initiate sperm activation. This process is spatially regulated because SWM-1, a protease inhibitor, suppresses TRY-5 activity in the seminal vesicle. That protease and protease inhibitors function in pair to modulate spermiogenesis was also demonstrated in Ascaris. Ascaris sperm are activated by the seminal fluid-derived protease As_TRY-5. MOs of activated sperm secrete the protease inhibitor As_SRP-1, which then forms a covalent complex with As_TRY-5, to block TRY-5 from activating other sperm. Possibly, the roles of a protease and a protease inhibitor in sperm activation have been evolutionarily conserved; e.g., in Drosophila a seminal fluid protease regulates post-mating reproductive processes (LaFlamme et al., 2012) and the lack of a serine protease inhibitor nexin-1 causes male fertility defects in mice (Murer et al., 2001).

Ascaris sperm activation in vitro requires a supply of bicarbonate/ $\mathrm{CO}_{2}$, as observed during sperm capacitation in mammals (Fraser, 2010). Whether in nematodes, bicarbonate also stimulates a soluble adenylate cyclase that generates cAMP as a second messenger for the downstream signaling cascade, as reported in flagellated sperm capacitation (Buck et al., 1999; Chen et al., 2000), deserves further investigations. Elucidating the regulatory mechanisms of nematode sperm activation might therefore provide new insights into the factors essential for male reproductive success and help to develop novel strategies for male contraception.

With respect to MSP-based sperm motility, progress has been made into the characterization of several phosphorylation-associated proteins (i.e., MPOP, MFPs, MPAK and $\mathrm{PP} 2 \mathrm{~A}$ ) in Ascaris. Additionally, the leading-edge protrusion and cell body retraction of Ascaris sperm have been reconstituted simultaneously in vitro (Shimabukuro et al., 2011).
Comparison of nematode sperm crawling with actin-based cell locomotion raises some interesting questions. For example, myosin, a motor protein, participates in eukaryotic cell motility; in contrast, MSP lacks detectable ATPase activity (Yi et al., 2009) and the energy source that powers nematode sperm motility remains elusive. Taken together, nematode sperm activation and motility are integrated events involving a complex signaling cascade that modulate cytoskeleton dynamics. This unique system could reveal some evolutionarily conserved mechanisms of cell motility.

\section{ACKNOWLEDGMENTS}

This research was supported by the National Basic Research Program of China (Nos. 2012CB94502, 2010CB912303) (to L.M.) and the National Natural Science Foundation of China (Grant Nos. 31171337 and 30971648 (to L.M.) and Grant Nos. 30871226 and 31071180 (to Y.Z.)). L.M. is supported by the Chinese Academy of Sciences 100-Talents Program. We thank Miss Wei Zhuang for excellent technical assistance.

\section{REFERENCES}

Abbas, M., and Cain, G.D. (1979). In vitro Activation and Behavior of Ameboid Sperm of Ascaris suum (Nematoda). Cell Tissue Res 200, 273-284.

Arduengo, P.M., Appleberry, O.K., Chuang, P., and L'Hernault, S.W. (1998). The presenilin protein family member SPE-4 localizes to an ER/Golgi derived organelle and is required for proper cytoplasmic partitioning during Caenorhabditis elegans spermatogenesis. J Cell Sci 111, 3645-3654.

Bandyopadhyay, J., Lee, J., II Lee, J., Yu, J.R., Jee, C., Cho, J.H., Jung, S., Lee, M.H., Zannoni, S., Singson, A., et al. (2002). Calcineurin, a calcium/calmodulin-dependent protein phosphatase, is involved in movement, fertility, egg laying, and growth in Caenorhabditis elegans. Mol Biol Cell 13, 3281-3293.

Buck, J., Sinclair, M.L., Schapal, L., Cann, M.J., and Levin, L.R. (1999). Cytosolic adenylyl cyclase defines a unique signaling molecule in mammals. Proc Natl Acad Sci U S A 96, 79-84.

Buttery, S.M., Ekman, G.C., Seavy, M., Stewart, M., and Roberts, T.M. (2003). Dissection of the Ascaris sperm motility machinery identifies key proteins involved in major sperm protein-based amoeboid locomotion. Mol Biol Cell 14, 5082-5088.

Chen, Y., Cann, M.J., Litvin, T.N., lourgenko, V., Sinclair, M.L., Levin, L.R., and Buck, J. (2000). Soluble adenylyl cyclase as an evolutionarily conserved bicarbonate sensor. Science 289, 625-628.

Edmonds, J.W., McKinney, S.L., Prasain, J.K., and Miller, M.A. (2011). The gap junctional protein INX-14 functions in oocyte precursors to promote C. elegans sperm guidance. Dev Biol 359, 47-58.

Findlay, G.D., Yi, X., MacCoss, M.J., and Swanson, W.J. (2008). Proteomics reveals novel Drosophila seminal fluid proteins transferred at mating. Plos Biol 6, 1417-1426.

Fraire-zamora, J.J., and Cardullo, R.A. (2010). The Physiological Acquisition of Ameboid Motility in Nematode Sperm: Is the Tail the 
Only Thing the Sperm Lost? Mol Reprod Dev 77, 739-750.

Fraser, L.R. (2010). The "switching on" of mammalian spermatozoa: molecular events involved in promotion and regulation of capacitation. Mol Reprod Dev 77, 197-208.

Geldziler, B., Chatterjee, I., and Singson, A. (2005). The genetic and molecular analysis of spe-19, a gene required for sperm activation in Caenorhabditis elegans. Dev Biol 283, 424-436.

Italiano, J.E., Roberts, T.M., Stewart, M., and Fontana, C.A. (1996). Reconstitution in vitro of the motile apparatus from the amoeboid sperm of Ascaris shows that filament assembly and bundling move membranes. Cell 84, 105-114.

Kubagawa, H.M., Watts, J.L., Corrigan, C., Edmonds, J.W., Sztul, E., Browse, J., and Miller, M.A. (2006). Oocyte signals derived from polyunsaturated fatty acids control sperm recruitment in vivo. Nature Cell Biol 8, 1143-U1183.

L'Hernault, S.W. (2006). Spermatogenesis. In Wormbook (The C. elegans Research Community).

L'Hernault, S.W. (2009). The genetics and cell biology of spermatogenesis in the nematode C. elegans. Mol Cell Endocrinol 306, 59-65.

LaFlamme, B.A., Ram, K.R., and Wolfner, M.F. (2012). The Drosophila melanogaster seminal fluid protease "seminase" regulates proteolytic and post-mating reproductive processes. Plos Genet 8. (In Press)

LeClaire, L.L., Stewart, M., and Roberts, T.M. (2003). A 48 kDa integral membrane phosphoprotein orchestrates the cytoskeletal dynamics that generate amoeboid cell motility in Ascaris sperm. J Cell Sci 116, 2655-2663.

Machaca, K., DeFelice, L.J., and Lhernault, S.W. (1996). A novel chloride channel localizes to Caenorhabditis elegans spermatids and chloride channel blockers induce spermatid differentiation. Dev Biol 176, 1-16.

Miao, L., Vanderlinde, O., Stewart, M., and Roberts, T.M. (2003). Retraction in amoeboid cell motility powered by cytoskeletal dynamics. Science 302, 1405-1407.

Minniti, A.N., Sadler, C., and Ward, S. (1996). Genetic and molecular analysis of spe-27, a gene required for spermiogenesis in Caenorhabditis elegans hermaphrodites. Genetics 143, 213-223.

Mogilner, A., and Oster, G. (2003). Cell biology. Shrinking gels pull cells. Science 302, 1340-1341.

Murer, V., Spetz, J.F., Hengst, U., Altrogge, L.M., de Agostini, A., and Monard, D. (2001). Male fertility defects in mice lacking the serine protease inhibitor protease nexin-1. Proc Natl Acad Sci U S A 98, 3029-3033.

Nance, J., Davis, E.B., and Ward, S. (2000). spe-29 encodes a small predicted membrane protein required for the initiation of sperm activation in Caenorhabditis elegans. Genetics 156, 1623-1633.

Nance, J., Minniti, A.N., Sadler, C., and Ward, S. (1999). spe-12 encodes a sperm cell surface protein that promotes spermiogenesis in Caenorhabditis elegans. Genetics 152, 209-220.

Nelson, G.A., and Ward, S. (1980). Vesicle fusion, pseudopod extension and ameboid motility are induced in nematode spermatids by the inophore monensin. Cell 19, 457-464.

Nishimura, H., and L'Hernault, S.W. (2010). Spermatogenesis-defective (spe) mutants of the nematode Caenorhabditis elegans provide clues to solve the puzzle of male germline functions during reproduction. Dev Dynamics 239,
1502-1514.

Park, B.J., Lee, D.G., Yu, J.R., Jung, S.K., Choi, K., Lee, J., Kim, Y.S., II Lee, J., Kwon, J.Y., Singson, A., et al. (2001). Calreticulin, a calcium-binding molecular chaperone, is required for stress response and fertility in Caenorhabditis elegans. Mol Biol Cell 12, 2835-2845.

Ram, K.R., and Wolfner, M.F. (2007). Seminal influences: Drosophila Acps and the molecular interplay between males and females during reproduction. Integrat Comp Biol 47, 427-445.

Reinke, V., Gil, I.S., Ward, S., and Kazmer, K. (2004). Genome-wide germline-enriched and sex-biased expression profiles in Caenorhabditis elegans. Development 131, 311-323.

Roberts, T.M., and King, K.L. (1991). Centripetal Flow and Directed Reassembly of the Major Sperm Protein (MSP) Cytoskeleton in the Ameboid Sperm of the Nematode, Ascaris suum. Cell Motil Cytoskel 20, 228-241.

Sepsenwol, S., and Taft, S.J. (1990). In vitro Induction of Crawling in the Ameboid Sperm of the Parasite, Ascaris suum. Cell Motil Cytoskel 15, 99-110.

Shakes, D.C., and Ward, S. (1989). Initiation of Spermiogenesis in C. elegans: a Pharmacological and Genetic Analysis. Dev Biol 134, 189-200.

Shimabukuro, K., Noda, N., Stewart, M., and Roberts, T.M. (2011). Reconstitution of Amoeboid Motility In Vitro Identifies a Motor-Independent Mechanism for Cell Body Retraction. Curr Biol 21, 1727-1731.

Singson, A. (2006). Sperm activation: Time and tide wait for no sperm. Curr Biol 16, R160-R162.

Smith, J.R., and Stanfield, G.M. (2011). TRY-5 Is a Sperm-Activating Protease in Caenorhabditis elegans Seminal Fluid. Plos Genet 7.

Stanfield, G.M., and Villeneuve, A.M. (2006). Regulation of sperm activation by SWM-1 is required for reproductive success of $C$. elegans males. Curr Biol 16, 252-263.

Vanfleteren, J.R., Van De Peer, Y., Blaxter, M.L., Tweedie, S.A.R., Trotman, C., Lu, L., Van Hauwaert, M.-L., and Moens, L. (1994). Molecular genealogy of some nematode taxa as based on cytochrome $\mathrm{c}$ and globin amino acid sequence. Mol Phylogenet Evol 3, 92-101.

Varkey, J.P., Jansma, P.L., Minniti, A.N., and Ward, S. (1993). The Caenorhabditis elegans Spe-6 Gene Is Required for Major Sperm Protein Assembly and Shows 2nd Site Noncomplementation with an Unlinked Deficiency. Genetics 133, 79-86.

Ward, S., Hogan, E., and Nelson, G.A. (1983). The initiation of spermiogenesis in the nematode Caenorhabditis elegans. Dev Biol 98, 70-79.

Washington, N.L., and Ward, S. (2006). FER-1 regulates $\mathrm{Ca}^{+}{ }^{+}$-mediated membrane fusion during $C$. elegans spermatogenesis. J Cell Sci 119, 2552-2562.

Wu, J.-C., Go, A.C., Samson, M., Cintra, T., Mirsoian, S., Wu, T.F., Jow, M.M., Routman, E.J., and Chu, D.S. (2012). Sperm development and motility are regulated by PP1 phosphatases in Caenorhabditis elegans. Genetics 190, 143-157.

Yi, K.X., Buttery, S.M., Stewart, M., and Roberts, T.M. (2007). A Ser/Thr kinase required for membrane-associated assembly of the major sperm protein motility apparatus in the amoeboid sperm of Ascaris. Mol Biol Cell 18, 1816-1825.

Yi, K.X., Wang, X., Emmett, M.R., Marshall, A.G., Stewart, M., and 
Roberts, T.M. (2009). Dephosphorylation of major sperm protein (MSP) fiber protein 3 by protein phosphatase 2a during cell body retraction in the MSP-based amoeboid motility of ascaris sperm. Mol Biol Cell 20, 3200-3208.

Zhao, Y., Sun, W., Zhang, P., Chi, H., Zhang, M.-J., Song, C.-Q., Ma, X., Shang, Y., Wang, B., Hu, Y., et al. (2012). Nematode sperm maturation triggered by protease involves sperm-secreted serine protease inhibitor (Serpin). Proc Natl Acad Sci U S A 109, 1542-1547.

Zhu, G.D., Salazar, G., Zlatic, S.A., Fiza, B., Doucette, M.M., Heilman, C.J., Levey, A.I., Faundez, V., and L'Hernault, S.W. (2009). SPE-39 family proteins interact with the HOPS complex and function in lysosomal delivery. Mol Biol Cell 20, 1223-1240. 